\title{
eJRIEPS
}

Ejournal de la recherche sur l'intervention en éducation physique et sport

42 | 2017

Varia

\section{Analyse de la dynamique temporelle d'un set en badminton en fonction de niveaux de pratique: réflexions sur les stratégies couramment proposées en EPS}

Dynamic Aspect of Temporal Structure according to Level of Expertise in Badminton: Analysis and Reflection on Teaching Practise in Physical Education

Olivier Dieu, Christophe Schnitzler, Elodie Drumez, Emilie Harmel et François Potdevin

\section{OpenEdition \\ Journals}

Édition électronique

URL : http://journals.openedition.org/ejrieps/542

DOI : $10.4000 /$ ejrieps.542

ISSN : $2105-0821$

Éditeur

ELLIADD

\section{Référence électronique}

Olivier Dieu, Christophe Schnitzler, Elodie Drumez, Emilie Harmel et François Potdevin, « Analyse de la dynamique temporelle d'un set en badminton en fonction de niveaux de pratique : réflexions sur les stratégies couramment proposées en EPS », eJRIEPS [En ligne], 42 | 2017, mis en ligne le 01 juillet 2017, consulté le 10 décembre 2020. URL : http://journals.openedition.org/ejrieps/542 ; DOI : https:// doi.org/10.4000/ejrieps.542

La revue eJRIEPS est mise à disposition selon les termes de la Creative Commons Attribution 4.0 International License. 


\section{eJRIEPS 42 juillet 2017}

\section{Analyse de la dynamique temporelle d'un set en badminton en fonction de niveaux de pratique: réflexions sur les stratégies couramment proposées en EPS}

Olivier Dieu 1, Christophe Schnitzler ${ }^{1}$, Elodie Drumez ${ }^{2}$, Emilie Harmel ${ }^{1}$ et François Potdevin ${ }^{1}$.

1 Unité de recherche Pluridiciplinaire Sport Santé Socitété (EA 7369), Univ. Lille, Univ. Artois, Univ. Littoral Côte d'Opale, EA 7369 - URePSSS - Unité de Recherche Pluridisciplinaire Sport Santé Société, F-59000 Lille, France.

2 Unité de Méthodologie Biostatistiques et Datamanagement du CHU de Lille, Univ. Lille, CHU Lille, Department of Biostatistics, EA 2694 - Santé publique : épidémiologie et qualité des soins, F-59000 Lille, France.

\section{Résumé}

Dans la littérature en badminton, l'aspect spatial prime sur l'aspect temporel. Les études sur la structure temporelle sont exclusivement descriptives, relatives au haut niveau, et produisent des données génériques et adynamiques, comme si le rapport au temps était le même pour l'ensemble des joueurs et sur l'ensemble du match. Notre étude vise à montrer que le rythme du set se réorganise avec les mobiles du badiste. 50 sujets, classés dans les cinq étapes du curriculum conatif du joueur de badminton ont porté un accéléromètre lors d'un set filmé. Nos résultats, portant sur l'analyse des 1806 points joués, montrent que le rapport temps d'échange / temps de non-jeu n'augmente pas de manière linéaire avec l'expertise et que le différentiel entre la quantité de mouvement en jeu et pendant les phases de repos se réorganise d'étape en étape. II y a donc des rythmes ou temporalités spécifiques en fonction des mobiles. Par ailleurs, au sein même de certaines étapes, on observe une variation de ces facteurs en fonction de l'évolution du rapport de force et / ou du moment du match pour les experts et les grands débutants. En pédagogie, c'est donc la spécificité du jeu qui devrait orienter le choix didactique entre jeu au temps / jeu au point afin d'envisager la temporalité de manière plus responsable.

Mots clés: badminton, temporalité, quantité d'activité physique, conation, stratégie responsable 


\section{eJRIEPS 42 juillet 2017}

\section{Introduction}

Le temps, quand il est évoqué dans l'activité badminton, est abordé selon trois angles:

un angle réglementaire, via les recommandations de la FFBAD concernant les temps de pauses et le principe de continuité du jeu ;

un angle de performance, via des recherches récentes, exclusivement descriptives et relatives au haut niveau comme le calcul des durées moyennes caractéristiques d'un match (Cabello Manrique \& Gonzales-Badillo, 2003 ; Phomsoupha \& Laffaye, 2014) ;

un angle didactique, via quelques articles relatifs à la gestion du rapport de force à travers le concept de pression temporelle exercée par l'un ou l'autre dans l'échange (Leveau, 2000 ; Preuvot, 2000).

Passé le cadre réglementaire, d'emblée, on note un contraste entre le foisonnement des recherches récentes sur la structure temporelle du match (Abia'n et al., 2014, Abian-vicen et al., 2013; Cabello \& Lees, 2004; Cabello Manrique \& González-Badillo, 2003; Chen \& Chen, 2008, 2011; Faude et al., 2007; Ming, Keong, \& Ghosh, 2008) et le peu d'ouvrages didactiques qui s'intéressent à la question du temps en raquette lors des vingt dernières années (Thibaut, 1993 ; Limouzin, 1998 ; Sève, 2000).

On remarque une sorte d'imperméabilité, à la fois du champ didactique, qui reste majoritairement cantonné à des problématiques d'ordre spatial, et des pratiques professionnelles qui occultent totalement la question en ayant recours à un jeu au temps quasi systématique.

Tout d'abord, au plan du règlement, le badminton repose sur un principe de continuité du jeu. Les arrêts réglementaires sont de deux minutes entre les sets et d'une minute à la moitié du set (11 points). Il est à noter cependant que la pratique de l'auto-arbitrage, majoritaire en badminton, laisse au joueur une certaine latitude sur ce principe de continuité du jeu car « en aucun cas le jeu ne sera suspendu pour permettre à un joueur de récupérer ses forces ou son souffle ou bien pour recevoir des conseils » (FFbad : Les règles officielles du badminton, septembre 2011).

En outre, l'aspect temporel dans la littérature professionnelle en sports de raquette est, à de rares exceptions près (Thibaut, 1993), largement minoré par rapport à l'aspect spatial. Une revue de littérature récente en badminton (Dieu, 2012) montre que les ouvrages et articles restent centrés sur l'espace comme en témoigne l'omniprésence des progressions relatives au volume de jeu ou «cartographie du terrain » en deux dimensions : droite / gauche et avant / arrière (Andréani, 1999 ; Estrabaud, Murignieux \& Tixier-Viriciel, 2000 ; Deconinck \& Fontaine, 2002) ou les cibles au sol à viser (Gauvin, 1995 ; Mérard \& Ventou, 


\section{eJRIEPS 42 juillet 2017}

1996 ; Vaufreydaz, 2000). Seuls quelques auteurs, minoritaires, reprennent l'idée de Thibaut (1993) selon laquelle : « le Badminton est davantage un jeu de temps qu'un jeu d'espace ». Ainsi, pour Ferly et Papelier (2003), si la classique distinction sur le volume de jeu (du centre vers les quatre coins) est reprise (p.79), s'y ajoute une vue latérale des trajectoires émises empruntées à Limouzin (1994, 2000) qui suggère un axe vertical de déplacement directement lié à une logique temporelle (en effet, le badiste saute souvent pour intercepter le volant et le jouer plus tôt). D'autres ouvrages évoquent la notion de «pression temporelle» (Leveau, 2000) définie comme «le rapport entre le temps disponible effectif et le temps requis » (Alain \& Proteau, 1978). L'aspect temporel est ici directement relié à l'aspect tactique puisqu'il est à la base de l'identification, par le joueur, de l'état du rapport de force (Pizzinato, 1993) : « Je domine si le temps disponible est supérieur au temps requis pour l'exécution de ma frappe, il s'agit alors de ne pas donner de temps à l'adversaire, ce qui implique de frapper le volant le plus tôt possible / Je suis dominé si le temps disponible est inférieur au temps requis, il s'agit alors de se donner du temps, notamment en relevant le volant afin de se replacer et d'être dans de bonnes conditions pour frapper ».

Néanmoins, si ces quelques articles dépassent une logique spatiale pure, l'aspect temporel n'est envisagé qu'à l'échelle du point (changement de statut dans l'échange), rarement dans l'évolution du match.

Dans les pratiques en milieu scolaire, l'activité badminton se joue souvent au temps (Voir à ce sujet, l'article de Laffaye (2006) dans lequel l'auteur compare les différentes formes d'organisation du badminton scolaire: montante-descendante, ronde italienne, tableau allemand et défis). La logique organisationnelle vient alors contrecarrer les préoccupations didactiques précédentes : «si la logique du jeu impose le déroulement d'un match en points, les exigences scolaires nous enjoignent à privilégier l'organisation au temps... plus efficace et fonctionnelle »(Leveau, 2007, p. 77). Ce jeu au temps a pourtant tendance à dénaturer l'activité-même. Le but du traditionnel match en montante-descendante (Descamps, 2008) est souvent de se « dépêcher» pour marquer le plus de points possibles. Dans ce cas, la question de la gestion du temps supplante celle de la gestion du score et modifie l'organisation temporelle du jeu. Le jeu « au temps », impose, de l'extérieur, un rythme soutenu dans la construction de l'échange et dans l'enchainement des points qui peut être préjudiciable à la construction de compétences.

En effet, à haut niveau, l'analyse de Laffaye et al. (2015) sur la finale olympique du simple homme à Londres en 2012 montre que la construction du point ne privilégie pas les points 


\section{eJRIEPS 42 juillet 2017}

courts $(20 \%$ des rallyes sont inférieurs à 3 sec., 25\% des rallyes sont compris entre 4 et 6 sec., $29 \%$ des rallyes sont compris entre 7 et 12 sec. et $26 \%$ des rallyes sont supérieurs à 13 sec.). Par ailleurs, l'enchainement des points n'est pas non plus métronomique, la durée du temps de non-jeu est corrélée à la quantité de frappes effectuées dans le rallye précédent (Laffaye et al, 2015). Enfin, la durée du non-jeu et significativement plus élevée chez les hommes que chez les femmes (Cabello et al., 2004 ; Abian-Vicen et al., 2013).

En définitive, le rythme du match, c'est-à-dire la perspective dynamique temporelle et énergétique de la mobilisation du joueur est, à de rares exceptions près (Sève, 2000), complétement occultée de la didactique actuelle en sport de raquette.

Pour preuve, le «non-jeu » ne fait jamais l'objet d'articles ou d'études. Dans l'esprit commun il semble ne pas faire partie du jeu... alors qu'il est (en badminton au moins) un outil précieux de gestion énergétique du match. Couartou \& Grunenfelder, dès 1992, pointaient cette spécificité de l'alternance: période de jeu / période de non-jeu en badminton par rapport au tennis ou au tennis de table: « Le badminton est très sollicitant en terme d'énergie, il est indispensable de ménager des temps de récupération » (p. 47). Ces auteurs mettaient déjà en garde contre une didactique qui minore le poids de l'action contextuelle au profit de simplifications évaluatives : «(..) si la recherche d'une rupture d'échange précoce peut être intéressante au tout début de la pratique, la tactique de base du badminton, qui est de construire le gain de l'échange en obligeant l'adversaire à se déplacer, à se fatiguer... est oubliée » (p. 148).

Enfin, au plan de la performance, de nombreuses études récentes s'intéressent à la question du temps en sport de raquette et notamment en badminton (Abia'n et al., 2014, Abian-Vicen et al., 2013; Cabello \& Lees, 2004; Cabello Manrique \& González-Badillo, 2003; Chen \& Chen, 2008, 2011; Faude et al., 2007; Ming, Keong, \& Ghosh, 2008). Néanmoins ces recherches sont incomplètes dans la mesure où elles restent descriptives, relatives au haut niveau et qu'elles ne prennent pas en compte la dynamique du set ou du match liée à l'évolution du rapport de force.

Ainsi, selon la revue de littérature de Phomsoupha \& Laffaye (2014) la durée moyenne d'un match est établie à $1885.08 \mathrm{sec}$. pour les simples hommes et à $1365.03 \mathrm{sec}$. pour les simples dames, avec des durées moyennes de $600 \mathrm{sec}$. par set joué. Les auteurs fixent la moyenne des temps de jeu (rally time) à $7.66 \mathrm{sec}$. chez les hommes et à $6.1 \mathrm{sec}$. chez les femmes ; la moyenne des périodes de non-jeu (rest time) à $15.4 \mathrm{sec}$. pour les hommes et à $14.0 \mathrm{sec}$. pour les femmes, et le temps effectif de jeu moyen (effective playing time) à $32.1 \%$ chez les hommes et $29.8 \%$ chez les femmes. 


\section{eJRIEPS 42 juillet 2017}

Ces données relatives, exclusivement au haut niveau, laissent à penser que la dynamique temporelle est une donnée stable. Or, rien ne laisse à penser que la structure temporelle en « badminton » est la même pour un novice, un débrouillé, un confirmé, un expert.

Notre première hypothèse est donc la suivante : le niveau d'expertise a un effet sur la structure temporelle.

De plus, une lecture plus fine montre que ces résultats ne sont pas homogènes, un simple à haut niveau durant de $40 \mathrm{~min}$. (Cabello Manrique \& González-Badillo, 2003) à 1 heure (Abia'n et al., 2014; Cabello \& Lees, 2004; Chen \& Chen, 2008). Selon Laffaye, Phomsouvah \& Dor (2015), le temps de jeu moyen est compris entre $4.6 \mathrm{sec}$. et $9.0 \mathrm{sec}$. et le temps de non jeu est compris entre $9.7 \mathrm{sec}$. et $24.1 \mathrm{sec}$. (Abian- Vicen et al., 2013; Cabello \& Lees, 2004; Cabello Manrique \& González-Badillo, 2003; Chen \& Chen, 2008; Faude et al., 2007; Ming, Keong \& Ghosh, 2008). Quant au temps de jeu effectif, il est compris entre $27.3 \pm 2.4 \%$ (Abian-Vicen et al., 2013) et $38.5 \pm 3.8 \%$ (Chen \& Chen, 2011). Ces différences peuvent s'expliquer par un ensemble de facteurs que nous qualifierons d' "exogènes".

Tout d'abord, les conditions de recueil des données ne sont pas toujours identiques : contrairement aux autres études précitées, les travaux de Faude et al. (2007) concernent des matchs simulés et non des matchs en conditions réelles de compétitions. Par ailleurs, on note de grandes variations en fonction de l'âge de la population experte (les travaux de Ming et al. portent sur des jeunes internationaux) mais aussi en fonction de l'époque à laquelle elles ont été réalisées. En effet, la forte augmentation relevée par Abia'n en 2013 (Rally $=9,0 \pm 0,9 \mathrm{sec}$. et Rest Time $=24,1 \pm 3,8 \mathrm{sec}$.), va dans le sens de l'étude longitudinale sur six finales olympiques masculines réalisée par Laffaye et al en 2015 (Rally $=10,1 \pm 0,7 \mathrm{sec}$. et Rest Time = 33,5 \pm 1,5 sec.). Depuis 1996, la durée des rallyes mais surtout la durée du non jeu n'a cessé d'augmenter, alors que le temps effectif de jeu lui a fortement baissé (-34,5\%). Laffaye \& Phomsouvah (2015) expliquent ces différences par le changement réglementaire de 2006 : on passe de 2 sets gagnants de 15 points où l'on ne pouvait marquer que sur son service, sans coaching autorisé entre les points, à 2 sets gagnants de 21 points en tie-break avec coaching autorisé entre les points et par un badminton « moderne », plus offensif, qui présente de plus en plus d'intensité dans le jeu. Néanmoins, certains travaux portant sur le même type de population et dans le même contexte de recueil de données aboutissent également à des données très hétérogènes, ce qui laisse à penser qu'il y a aussi des facteurs "endogènes" à ces différences de "temporalités". 


\section{eJRIEPS 42 juillet 2017}

Liddle \& O'Donoghue (1998) s'intéressent aux caractéristiques du badminton féminin et masculin durant la saison 1996 : la durée des rallyes est comprise entre 6.35 et $9.15 \mathrm{sec}$. et les temps de non-jeu entre 11.03 et $15.01 \mathrm{sec}$. Une analyse du même tournoi un an plus tard (O'Donoghue, 2000) montre des différences significatives entre les deux saisons, particulièrement la moyenne des rallyes masculins qui passe de $9.15 \mathrm{sec}$. à $6.70 \mathrm{sec}$. Plus récemment, Pritchard et al. (2001), obtiennent des moyennes de rallyes masculins sur les Welsh Open de 1999 et 2000 (conditions similaires de jeu), respectivement de 7.27 et $8.11 \mathrm{sec}$. Les résultats de Cabello et Gonzales-Badillo, 2003 montrent des durées de rallyes autour de $6.40 \mathrm{sec}$. Ces données montrent des variabilités importantes dans le jeu entre des tournois élites dans un contexte pourtant similaire (avant le changement de règlement) et sur un laps de temps très restreint (1998-2003).

Par ailleurs, certains chercheurs ont essayé de mettre en relation temps de jeu et nonjeu et là aussi on note une forte variabilité dans les résultats obtenus. Cabello Manrique \& Gonzales-Badillo (2003) montrent une forte corrélation $(r=0.87)$ entre le temps de jeu et le temps de non jeu suivant. Ainsi, pour les auteurs, un échange long nécessiterait une récupération plus longue, afin de recouvrer un seuil cardiorespiratoire favorable à l'engagement dans un nouveau rallye (Fahimi \& Vaezmousavi, 2011). Néanmoins, les travaux de Laffaye et Phomsoupha (2015) viennent nuancer les conclusions de Cabello et al. avec une corrélation bien plus faible $(r=0.38 ; p<0.01)$ entre le temps de jeu et le temps de non-jeu suivant. Ce résultat laisse à penser que la seule raison physiologique n'est pas suffisante pour expliquer la durée du temps de non-jeu. L'hypothèse des auteurs est que la différence est aussi probablement due à une question tactique, le temps de non-jeu servant aussi à penser au point suivant seul, ou à échanger quelques mots avec le coach, ce que permet désormais le nouveau règlement depuis 2006. Cependant les travaux de Liddle \& O' Donoghue (1998) sur l'analyse des matchs du circuit européen contredisent les résultats de Cabello et al. en montrant que le rallye le plus long est associé à la plus courte phase de non-jeu...

L'hétérogénéité des données recueillies pour une population relativement homogène (celle des experts) laisse à penser que la structure temporelle du match de badminton varie selon des facteurs plus intrinsèques au jeu comme le rapport de force et son évolution (état du score) mais aussi le moment du match (début ou fin de set). C'est notre hypothèse 2 . 


\section{eJRIEPS 42 juillet 2017}

De plus, ces données temporelles, descriptives, ne sont qu'à de rares exceptions près (Laffaye et al., 2015) reliées directement à la nature du point joué. Or, la question du temps peut-elle être envisagée ex nihilo, indépendamment de celle de l'intensité du jeu ? Tout se passe finalement comme si, aux plans réglementaires, didactiques et de la performance, la composante temporelle de la mobilisation du joueur était une donnée générique à l'ensemble des joueurs, indépendante de la composante énergétique et adynamique en fonction de l'état du rapport de force et du moment du match.

Or, l'observation de joueurs in situ suggère différentes manières de gérer son temps en contexte de match. En effet, certains joueurs se précipitent pour ramasser le volant et le remettre aussitôt en jeu, quand d'autres prennent leur temps pour servir ou demandent à changer le volant à 18 égalité dans le set décisif, afin de marquer une pause... Ainsi, malgré le cadre réglementaire, l'observation de joueurs de niveaux différents nous invite à penser que le rythme du jeu, c'est-à-dire la combinaison de la durée et de l'intensité des périodes de jeu et de non-jeu, n'est pas le même selon l'expérience du joueur.

L'approche conative (Bui-Xuân, 1998) se fonde sur l'idée que la progression en activité sportive ne se résume pas à une simple augmentation du niveau de performance mais à un changement d'étape conative, c'est-à-dire un changement qualitatif du sens de la mobilisation du joueur. Celui-ci mobilise, en fonction de son expérience (étape conative), de façon prévalente, soit sa structure (capacités physiques), soit sa technique (habiletés motrices), soit sa fonctionnalité (aspects décisionnels ou choix tactiques).

L'approche conative est basée sur une classification qualitative qui modélise, d'une part le sens profond de l'activité, son " principe directeur » et, d'autre part l'évolution du sens que chaque individu confère à son action en fonction de son expérience dans l'activité (le curriculum conatif). Le sens de l'activité, son principe directeur, représente la relation intime qui lie les trois dimensions: structurale, technique et fonctionnelle, la part de chacune par rapport aux autres, leurs proportions, leur contribution à l'exécution de la prestation » (Bui-Xuân, 1994, p. 79). Par exemple le badminton, comme tous les sports de raquette (et d'opposition en général), est une activité à dominante clairement fonctionnelle, mais en proportion, elle est techniquement moins exigeante que le tennis mais structuralement plus exigeante que le tennis de table (Lees, 2003). Le sens du sujet évolue lui en fonction de son expérience de jeu à travers cinq étapes conatives du débutant à l'expert. Chaque étape se définit par la prévalence conative du joueur en fonction de son vécu dans l'activité. A l'étape 1, la prévalence est structurale, à l'étape 2, la prévalence est fonctionnelle, à l'étape 3 : la prévalence est technique. L'étape 4 se 


\section{eJRIEPS 42 juillet 2017}

caractérise par une prévalence technico-fonctionnelle. Enfin, à l'étape 5, d'expertise, les prévalences sont, à la fois, structurales, fonctionnelles et techniques. Ce curriculum conatif et les critères de classification aux différentes étapes ont fait l'objet d'utilisations dans des études récentes sur la pratique sportive (Vanhelst et al., 2012a) et notamment en badminton (Dieu, 2012). Le tableau I les répertorie.

Tableau 1. Description du curriculum conatif et des indicateurs en Badminton

\begin{tabular}{|c|c|c|c|}
\hline Etapes conatives & Description du comportement & Indicateurs de classement & Prévalence tactique \\
\hline $\begin{array}{l}\text { Etape } 1 \\
\text { Structurale } \\
\text { Conation = } \\
\text { Renvoyer }\end{array}$ & $\begin{array}{l}\text { Le joueur est relanceur, il repousse le } \\
\text { volant de l'autre côté du filet. Le volant } \\
\text { est pour lui un danger dont il doit se } \\
\text { débarrasser. }\end{array}$ & $\begin{array}{l}\text { Renvois longs axe central. Pas de } \\
\text { différenciation de statut d'attaquant ou } \\
\text { défenseur. }\end{array}$ & - \\
\hline $\begin{array}{l}\text { Etape } 2 \\
\text { Fonctionnelle } \\
\text { Conation = Diriger }\end{array}$ & $\begin{array}{l}\text { Le joueur est un placeur, il cherche à } \\
\text { diriger le volant dans l'espace libre. }\end{array}$ & $\begin{array}{l}\text { Variations de trajectoires et apparition } \\
\text { des statuts. Le dominé court après le } \\
\text { volant. Le dominant tente d'envoyer le } \\
\text { volant du côté où l'adversaire n'est pas. }\end{array}$ & ++ \\
\hline $\begin{array}{l}\text { Etape } 3 \\
\text { Technique } \\
\text { Conation = Placer } \\
\text { un coup gagnant }\end{array}$ & $\begin{array}{l}\text { Le joueur est un technicien (smash, } \\
\text { amorti, dégagement), qui « se regarde } \\
\text { dans l'exécution de la frappe » et tarde } \\
\text { donc à se replacer. }\end{array}$ & $\begin{array}{l}\text { Dégagement ou Smash en attaque. } \\
\text { Temps d'arrêt dans le replacement pour } \\
\text { observer le résultat de son action. }\end{array}$ & - \\
\hline $\begin{array}{l}\text { Etape } 4 \\
\text { Contextuelle } \\
\text { Conation = } \\
\text { Enchaîner }\end{array}$ & $\begin{array}{l}\text { Le joueur est tacticien : il enchaine les } \\
\text { frappes dans une séquence de jeu. II } \\
\text { joue avec le temps (intercepte en } \\
\text { attaque / relève le volant en défense) }\end{array}$ & $\begin{array}{l}\text { Replacement orienté après la frappe } \\
\text { (enchaînements) et apparition du jeu en } \\
\text { interception. }\end{array}$ & ++ \\
\hline $\begin{array}{l}\text { Etape } 5 \\
\text { Expertise } \\
\text { Conation = Imposer } \\
\text { son style de jeu }\end{array}$ & Le joueur est expert. & $\begin{array}{l}\text { Mobilise sa structure propre et ses } \\
\text { techniques selon son profil de jeu } \\
\text { (« rameur », « attaquant », «prendre le } \\
\text { filet »...) pour construire le point. }\end{array}$ & $++t$ \\
\hline
\end{tabular}

A l'étape 1 (structurale), la question première du débutant est la suivante : comment se débarrasser du volant ? Avant de « gagner », la préoccupation est de « ne pas perdre »; l'intention du joueur est alors de repousser le plus fort possible le volant dans le cours adverse, c'est pourquoi les capacités physiques sont les premières mobilisées. A partir du moment où le joueur a réglé le problème du renvoi, se pose alors la question suivante : comment battre l'adversaire ? Dès lors il commence à jouer dans les espaces libres pour faire bouger son adversaire. A cette étape 2, dite fonctionnelle, les ressources décisionnelles sont prioritairement mobilisées. A l'étape 3 se pose la question de l'efficacité : comment font ceux qui gagnent ? Le joueur entre dans une étape technique : il perd en fonctionnalité ce qu'il gagne en efficacité technique car il est préoccupé par le beau geste, l'exécution du mouvement. Ce n'est qu'à l'étape 4, contextuelle, que le joueur est capable de contextualiser la technique dans un enchaînement d'actions. A cette étape, 


\section{eJRIEPS 42 juillet 2017}

la prévalence tactique émerge à nouveau : la mobilisation du joueur est à la fois technique et fonctionnelle. Enfin, l'étape d'expertise (étape 5), se caractérise par une mobilisation optimale des ressources structurales, fonctionnelles et techniques du joueur pour fonder un style de jeu.

Bui-Xuân $(1998,1999)$ montre ainsi que la pensée tactique ou fonctionnalité est déjà caractéristique du niveau 2 (étape qualifiée de fonctionnelle), diminue en étape 3 (étape qualifiée de technique) pour redevenir prioritaire au niveau 4 (étape dite contextuelle).

Nous reprendrons donc à notre compte, à travers le modèle conatif (Bui-Xuân, 1999 ; Vanhelst, 2012), cette classification qualitative de l' "expérience du joueur » afin de ne pas limiter nos résultats à la seule population experte. De plus, dans le cadre de cette étude, nous n'envisagerons pas la question du temps ex nihilo mais en relation étroite avec l'intensité du jeu à travers la notion de rythme de jeu afin de tester les hypothèses suivantes.

$\mathrm{H} 1$ : le rythme du set (intensité et durée des périodes de jeu et de non-jeu) évolue en fonction de l'expérience du joueur (ses conations).

H2 : Au sein d'une même étape : le rythme du set évolue en fonction de l'état du rapport de force et / ou du moment du match.

\section{Méthode}

\section{1. Participants}

Quarante adolescents et adultes sains âgés de 17 à 37 ans ont participé à cette étude. Ils étaient tous volontaires, soit étudiants, soit joueurs de badminton affiliés à la FFBAD (31 hommes et 9 femmes). Leurs caractéristiques sont détaillées dans le tableau II.

Tableau 2. Caractéristiques des sujets.

\begin{tabular}{cccc}
\hline & Total & Hommes & Femmes \\
\hline $\mathrm{N}$ & 40 & 31 & 9 \\
Age (années) & $22 ; 20[17-37]$ & $22 ; 20[17-37]$ & $21 ; 20[18-26]$ \\
Taille $(\mathrm{cm})$ & $176 ; 176[160-195]$ & $178 ; 178[160-195]$ & $168 ; 168[162-176]$ \\
Poids $(\mathrm{kg})$ & $70.0 ; 69.5[52.0-100.0]$ & $73.0 ; 73.0[53.0-100.0]$ & $59.4 ; 58.0[52.0-70.0]$
\end{tabular}




\section{eJRIEPS 42 juillet 2017}

Les sujets ont joué plusieurs sets contre des adversaires différents. Ils ont tous été filmés et classés par niveau d'expérience $(1,2,3,4,5)$ à l'aide des critères du curriculum conatif (Bui-Xuân, 1999) appliqué à la pratique du Badminton (Dieu, 2010) (tableau I). Les données conservées ne pouvaient être retenues à l'avance. En effet, afin d'avoir des rapports de force homogènes a priori, nous confrontions systématiquement des joueurs de même étape. Néanmoins, l'opposition réelle traduisait parfois des résultats déséquilibrés. Ainsi pour que les données soient conservées nous avons estimé qu'il fallait que le score du perdant du set soit supérieur à 11 points. A cette condition le rapport de force était jugé équilibré dans la mesure où le perdant marquait plus de la moitié des points nécessaires à la victoire du set.

\section{2. Outils}

\section{2. 1. Classification des sujets dans les étapes conatives}

La classification théorique des étapes conatives (tableau I) a été validée grâce à un test vidéo proposé à dix spécialistes de badminton qui composait le jury du concours national de recrutement des professeurs d'éducation physique en France en 2011. Ce test interactif consistait en deux phases : une présentation vidéo de la classification conative en badminton (étapes conatives et indicateurs macroscopiques pour chaque étape) suivie d'une présentation de seize vidéos où les jurys devaient entourer l'étape correspondant au joueur et préciser l'indicateur correspondant. Le taux de validité du test est satisfaisant puisqu'il « révèle une marge de $5 \%$ d'erreur pour des jurys d'âge, de région et donc de formation initiale très différentes » (Dieu, 2015). De plus, contrairement au jury qui n'avait que six minutes de visionnage dans l'étude précitée, nous avons fonctionné sur des observations longues, doublées d'enregistrements vidéo qui ont permis une ou plusieurs observations a posteriori pour écarter les vidéos qui pouvaient être litigieuses.

Lors des observations préalables, si les indicateurs macroscopiques étaient repérés, nous classions l'individu à l'étape correspondante. Lors des tests, nous confrontions systématiquement deux joueurs de même étape.

Par le jeu des oppositions entre nos 40 sujets, nous avons pu conserver 50 sets joués à rapport de force homogène, et recueillir ainsi 1806 données en termes de points joués. (tableau III). 


\section{eJRIEPS 42 juillet 2017}

Tableau 3. Nombre de rallyes joués par étape conative.

\begin{tabular}{lcccccccc}
\hline & Sets & Hommes & Femmes & Rallyes $(\mathrm{N})$ & Court (0-3s) & Moyen (4-7s) & Long (8-11s) & très long (>11s) \\
\hline Etape structurale & 10 & 4 & 6 & 372 & 105 & 161 & 62 & 44 \\
Etape fonctionnelle & 10 & 10 & 0 & 373 & 89 & 208 & 65 & 11 \\
Etape technique & 10 & 6 & 4 & 347 & 50 & 233 & 52 & 12 \\
Etape contextuelle & 10 & 10 & 0 & 372 & 61 & 209 & 66 & 36 \\
Etape d'expertise & 10 & 10 & 0 & 342 & 41 & 125 & 90 & 86 \\
\hline
\end{tabular}

2. 2. 2. Classification des données en termes de dynamique de set

Disposant des vidéos, nous avons voulu investiguer la dynamique de jeu point par point, pour étudier comment le joueur, en fonction de son étape, gérait l'alternance jeu / non-jeu en termes de temps mais aussi en termes de quantité de mouvements. De plus nous avons séquencé les points joués en fonction de l'état du rapport de force : nous avons considéré le joueur dominant quand il mettait 3 points consécutifs, dominé quand il perdait 3 points consécutifs, pour le reste des points nous avons considéré le rapport de force neutre. Enfin, nous avons isolé les données du joueur en fin de set (après 15 points).

2. 2. 3. Mesure de la structure temporelle (Durée)

Les enregistrements vidéo des matchs nous ont permis d'isoler la durée des temps de jeu et de non-jeu pour chaque point joué. La variable dépendante retenue dans cette étude est le rapport entre le temps de jeu et le temps de non-jeu, puisque dans une perspective dynamique de gestion, le temps de non-jeu n'a de sens que par rapport au temps de jeu qui précède. Le rapport du temps en jeu sur le temps en non-jeu traduit le différentiel entre la durée passée dans l'échange et la durée passée en dehors de l'échange pour chaque point joué. Si ce rapport est supérieur à 1 le temps de non-jeu est plus court que le temps de jeu, si ce rapport est inférieur à 1 , le temps de non-jeu est plus long que le temps de jeu. La moyenne de ces données par étape est répertoriée dans le tableau 4.

2. 2. 4. Mesure de l'activité physique (Intensité)

Tous les participants ont porté un accéléromètre pendant un set de badminton (21 points en « tie-break » sans prolongation). La seule consigne était de jouer « comme en match » en portant une ceinture élastique sur laquelle est fixé l'accéléromètre GT3X situé dans le bas du dos au niveau de la colonne vertébrale, au contact de la peau (Figure 1). 


\section{eJRIEPS 42 juillet 2017}
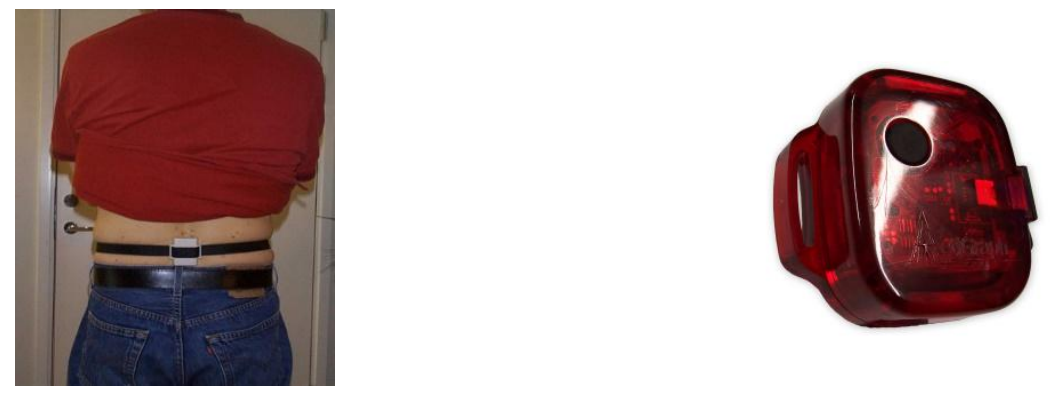

Figure 1. Pose de l'accéléromètre

Nous avons eu recours à l'accélérométrie (Bailey, et al., 1995; Craig, et al., 2003; De Jonge, et al., 2007) pour quantifier l'activité physique (Chen \& Bassett, 2005 ; Chu, McManus, \& Yu, 2007 ; Rowlands, et al., 2004 ; Treuth, et al., 2004 ; Vanhelst, et al., 2010). Cette méthode de mesure objective et non invasive de l'activité physique présente l'avantage de pouvoir être utilisée dans les conditions habituelles de vie (Vanhelst, et al., 2009, 2012b, 2012c, 2012d). C'est donc une méthode de choix qui est applicable en recherche clinique ou épidémiologique pour des études de physiologie mais qui peut l'être aussi en pédagogie puisqu'elle permet une observation en condition réelle de jeu (Dieu, et al., 2014 ; Silva, et al., 2015).

L'accéléromètre utilisé dans cette étude (GT3X®, Pensacola, FL, USA) mesure l'activité physique sur trois axes (vertical, antéropostérieur, et médio-latéral). La quantité d'activité physique est alors calculée grâce au vecteur magnitude (VM) qui est la racine carrée de la somme des counts enregistrés sur chaque vecteur ( $X, Y$ et $Z$ ). L'appareil a été calibré selon les spécifications du constructeur et paramétré à la seconde.

La variable dépendante retenue dans cette étude est le rapport entre le vecteur magnitude en jeu et le vecteur magnitude en non-jeu, puisque dans une perspective dynamique de gestion, la quantité de mouvement d'une période de non-jeu n'a de sens que par rapport à la quantité de mouvement de la période de jeu qui précède. Ce rapport traduit le différentiel entre la quantité de mouvement engagée dans l'échange et la quantité de mouvement engagée en dehors de l'échange pour chaque point joué. Plus ce rapport est supérieur à 1, plus la quantité de mouvement engagée dans le non-jeu est faible par rapport à la quantité engagée en jeu. La moyenne de ces données par étape est répertoriée dans le tableau $\mathrm{V}$.

2. 3. Protocole

L'étude s'est déroulée sur une période allant du 13 janvier au 26 avril 2012. Au début du créneau d'entrainement dans lequel se déroulaient les mesures, les sujets repérés en 


\section{eJRIEPS 42 juillet 2017}

amont comme appartenant à la même étape conative étaient informés de la possibilité d'être mis à contribution pour une étude sur le badminton. Pour ne pas affecter la validité interne de l'étude, la thématique de l'approche conative n'était pas exposée. Les sujets volontaires se voyaient alors remettre un document rassemblant une lettre d'information et une fiche de consentement. Le protocole était le suivant : il s'agissait de jouer un set de badminton de 21 points sans prolongation, ni pause à la moitié du set, contre un adversaire imposé en portant une ceinture accélérométrique sur laquelle est fixé l'actimétre GT3X (positionnement de l'appareil en bas du dos au niveau de la colonne vertébrale). Les participants étaient filmés mais pour que l'influence de l'appareillage soit minime, le caméscope était placé en début de séance derrière un terrain afin que sa présence soit démystifiée.

Grâce à ce protocole et par le jeu des oppositions entre nos 40 sujets, nous avons pu recueillir 50 sets joués à rapport de force homogène, ce qui aboutit à 1806 données en termes de rallyes joués. (tableau III).

\section{4. Analyses statistiques}

Les données ont été décrites en termes de moyenne ; médiane [étendue] pour les variables quantitatives. Compte tenu que la plupart des variables quantitatives sont des variables discrètes avec des distributions asymétriques, des tests non paramétriques ont été réalisés. Le rythme du match, à savoir la durée et l'intensité, a été comparé entre les étapes conatives et entre les rapports de force au sein de chaque étape (sur le set total puis uniquement sur les valeurs en fin de set) à l'aide du test de Kruskal-Wallis. Les comparaisons post-hoc deux à deux entre chaque étape conative successive ont été réalisées par un test du $U$ de Mann-Whitney en appliquant la correction de Bonferroni pour comparaisons multiples. Les corrélations entre le temps de jeu et le temps de non-jeu en fonction des étapes conatives au global puis selon les durées des échanges ont été étudiées à l'aide du coefficient de corrélation des rangs de Spearman. Le niveau de significativité des tests a été fixé à $5 \%$. Les données ont été analysées en utilisant le logiciel SAS version 9.4 (SAS Institute, Cary, NC).

\section{Résultats}

3. 1. Résultats globaux (influence de l'étape sur le rythme du match)

3. 1. 1. Concernant la durée

La distribution du rapport du temps en jeu sur le temps en non-jeu ainsi que ses composantes est décrite par étape dans le tableau 4 et sur la figure 2. Le rapport jeu / 


\section{eJRIEPS 42 juillet 2017}

non-jeu diffère significativement entre les 5 étapes conatives $(p<0.05)$. Les comparaisons post-hoc deux à deux montrent que l'étape 4 a un rapport plus élevé que l'étape 3 ( $p<$ 0.05 ) et moins élevé que l'étape 5 ( $p<0.05)$. Ainsi, le temps de non-jeu diminue significativement à l'étape 4 et augmente significativement à l'étape 5 . Concernant les corrélations entre la durée du temps de jeu et la durée du repos qui suit (tableau 5), aucune relation significative n'a été retrouvée, exceptée pour l'ensemble des échanges de l'étape 3 où une relation négative faible est ressortie significative (rho $=-0.14, p=0.009$ ).

Tableau 4. Distribution de la durée en secondes pour chaque étape conative

\begin{tabular}{ccccccc}
\hline \multicolumn{7}{c}{ Etapes } \\
\hline & Structurale & Fonctionnelle & Technique & Contextuelle & Expertise & $p$ \\
\hline Temps de jeu & $6.4 ; 5.0$ & $5.5 ; 5.0$ & $5.7 ; 5.0$ & $6.3 ; 5.0$ & $9.4 ; 8.0$ & $<0.0001$ \\
& {$[1.0 ; 24.0]$} & {$[2.0 ; 18.0]$} & {$[1.0 ; 19.0]$} & {$[2.0 ; 20.0]$} & {$[1.0 ; 42.0]^{*}$} & \\
Temps de non & $7.2 ; 7.0$ & $6.6 ; 6.0$ & $7.0 ; 7.0$ & $6.7 ; 6.0$ & $10.7 ; 9.5$ & \\
jeu & {$[1.0 ; 19.0]$} & {$[1.0 ; 17.0]^{*}$} & {$[2.0 ; 14.0]$} & {$[2.0 ; 20.0]^{*}$} & {$[1.0 ; 42.0]^{*}$} & $<0.0001$ \\
Rapport jeu / & $0.95 ; 0.80$ & $0.86 ; 0.78$ & $0.89 ; 0.75$ & $1.02 ; 0.83$ & $0.92 ; 0.74$ & \\
non-jeu & {$[0.00 ; 4.75]$} & {$[0.00 ; 3.00]$} & {$[0.00 ; 4.00]$} & {$[0.14 ; 3.0]^{*}$} & {$[0.05 ; 7.50]^{*}$} & 0.0022 \\
\end{tabular}

Les données sont présentées en moyenne ; médiane [étendue].

${ }^{*} p<0.05$ pour les comparaisons post-hoc avec l'étape conative précédente.

Tableau 5. Corrélation entre le temps de jeu et le temps de non-jeu en fonction des étapes conatives et des durées des échanges.

\begin{tabular}{|c|c|c|c|c|c|c|c|c|c|c|}
\hline & \multicolumn{2}{|c|}{ Structurale } & \multicolumn{2}{|c|}{ Fonctionnelle } & \multicolumn{2}{|c|}{ Technique } & \multicolumn{2}{|c|}{ Contextuelle } & \multicolumn{2}{|c|}{ Expertise } \\
\hline & Rho & $\mathrm{P}$ & Rho & $P$ & Rho & $P$ & Rho & $P$ & Rho & $\mathrm{P}$ \\
\hline Global & 0.09 & 0.07 & -0.02 & 0.66 & -0.14 & 0.009 & -0.01 & 0.93 & 0.11 & 0.052 \\
\hline Points courts & 0.14 & 0.14 & -0.10 & 0.34 & 0.09 & 0.55 & -0.23 & 0.07 & -0.13 & 0.43 \\
\hline Points moyens & 0.12 & 0.12 & -0.12 & 0.10 & -0.09 & 0.15 & 0.02 & 0.77 & 0.16 & 0.07 \\
\hline Points longs & -0.17 & 0.19 & 0.08 & 0.53 & -0.07 & 0.62 & -0.06 & 0.64 & -0.02 & 0.87 \\
\hline Points très longs & -0.12 & 0.45 & 0.06 & 0.87 & -0.21 & 0.51 & -0.16 & 0.36 & -0.05 & 0.62 \\
\hline
\end{tabular}




\section{eJRIEPS 42 juillet 2017}

\section{1. 1. Concernant l'intensité}

Comme montré dans le tableau VI, le rapport VM jeu / non-jeu diffère significativement entre les 5 étapes $(p<0,0001)$. Le rapport VM jeu / non-jeu augmente avec le niveau des étapes conatives avec des différences deux à deux significatives excepté entre les étapes 2 et 3 . On observe particulièrement une augmentation très nette du différentiel jeu / nonjeu de l'étape contextuelle (4) et à l'étape d'expertise (5).

Tableau 6. Distribution de l'intensité en counts pour chaque étape conative.

\begin{tabular}{ccccccc}
\hline & & \multicolumn{2}{c}{ Etapes } & & \\
\hline & Structurale & Fonctionnelle & Technique & Contextuelle & Expertise & $p$ \\
\hline VM en jeu & $148.1 ; 145.1$ & $172.9 ; 178.7$ & $176.5 ; 184.1$ & $232.0 ; 238.8$ & $266.0 ; 274.5$ & $<0.0001$ \\
& {$[47.3 ; 262.6]$} & {$[12.1 ; 282.8]^{*}$} & {$[47.2 ; 286.4]$} & {$[68.2 ; 373.9]^{*}$} & {$[29.7 ; 377.6]^{*}$} & \\
\hline VM en non jeu & $93.8 ; 90.8$ & $96.6 ; 98.7$ & $94.6 ; 93.7$ & $88.7 ; 81.9$ & $81.6 ; 59.8$ & $<0.0001$ \\
& {$[25.9 ; 219.1]$} & {$[37.7 ; 188.9]$} & {$[20.7 ; 189.5]$} & {$[24.5 ; 213.7]^{*}$} & {$[23.3 ; 336.5]^{*}$} & \\
\hline VM jeu / VM non-jeu & $1.77 ; 1.59$ & $2.03 ; 1.86$ & $2.25 ; 1.85$ & $3.07 ; 2.89$ & $4.88 ; 4.56$ & $<0.0001$
\end{tabular}

Les données sont présentées en moyenne ; médiane [étendue].

${ }^{*} \mathrm{p}<0.05$ pour les comparaisons post-hoc avec l'étape conative précédente.

La norme du vecteur magnitude (VM), exprimée par la formule suivante : VM $=\sqrt{ } x^{2}+y^{2}+z^{2}$, nous renseigne sur la quantité de mouvement ou dépense énergétique en counts par seconde.

3. 2. Résultats détaillés (influence du rapport de force et du moment du match pour chaque étape)

3. 2. 1. Concernant la durée 
eJRIEPS 42 juillet 2017

Tableau 6. Distribution du rapport de temps de jeu / non-jeu par étape en fonction du rapport de force.

\begin{tabular}{|c|c|c|c|c|c|c|c|c|c|}
\hline \multicolumn{7}{|c|}{ Rapport de force durant le set } & \multicolumn{3}{|c|}{ Rapport de force en fin de set } \\
\hline Etapes & Global & Dominant & Neutre & Dominé & $P$ & Dominant & Neutre & Dominé & $P$ \\
\hline Structurale & $\begin{array}{c}0.95 ; 0.80 \\
{[0.00 ; 4.75]}\end{array}$ & $\begin{array}{c}1.01 ; 0.83 \\
{[0.00 ; 4.75]}\end{array}$ & $\begin{array}{c}0.89 ; 0.77 \\
{[0.00 ; 4.00]}\end{array}$ & $\begin{array}{l}1.01 ; 0.83 \\
{[0.00 ; 4.75]}\end{array}$ & NS & $\begin{array}{c}0.88 ; 0.75 \\
{[0.13 ;} \\
3.00]\end{array}$ & $\begin{array}{c}0.74 ; 0.56 \\
{[0.22 ;} \\
2.17]\end{array}$ & $\begin{array}{c}0.88 ; 0.75 \\
{[0.13 ;} \\
3.00]\end{array}$ & NS \\
\hline Fonctionnelle & $\begin{array}{c}0.86 ; 0.78 \\
{[0.00 ; 3.00]}\end{array}$ & $\begin{array}{l}0.81 ; 0.75 \\
{[0.00 ; 2.00]}\end{array}$ & $\begin{array}{c}0.88 ; 0.77 \\
{[0.00 ; 3.00]}\end{array}$ & $\begin{array}{c}0.81 ; 0.75 \\
{[0.00 ; 2.00]}\end{array}$ & NS & $\begin{array}{c}0.92 ; 0.83 \\
{[0.38 ;} \\
2.00]\end{array}$ & $\begin{array}{c}1.09 ; 1.00 \\
{[0.22 ;} \\
3.00]\end{array}$ & $\begin{array}{c}0.92 ; 0.83 \\
{[0.38 ;} \\
2.00]\end{array}$ & NS \\
\hline Technique & $\begin{array}{c}0.89 ; 0.75 \\
{[0.00 ; 4.00]}\end{array}$ & $\begin{array}{l}0.81 ; 0.75 \\
{[0.18 ; 1.80]}\end{array}$ & $\begin{array}{c}0.93 ; 0.78 \\
{[0.00 ; 2.80]}\end{array}$ & $\begin{array}{c}0.76 ; 0.75 \\
{[0.18 ; 1.43]}\end{array}$ & NS & $\begin{array}{c}0.73 ; 0.69 \\
{[0.18 ;} \\
1.43]\end{array}$ & $\begin{array}{c}0.85 ; 0.75 \\
{[0.25 ;} \\
2.17]\end{array}$ & $\begin{array}{c}0.65 ; 0.67 \\
{[0.18 ;} \\
1.00]\end{array}$ & NS \\
\hline Contextuelle & $\begin{array}{c}1.02 ; 0.83 \\
{[0.14 ; 3.00]}\end{array}$ & $\begin{array}{c}1.00 ; 0.86 \\
{[0.14 ; 2.67]}\end{array}$ & $\begin{array}{c}1.03 ; 0.83 \\
{[0.25 ; 3.00]}\end{array}$ & $\begin{array}{c}1.00 ; 0.86 \\
{[0.14 ; 2.67]}\end{array}$ & NS & $\begin{array}{c}0.84 ; 0.80 \\
{[0.33 ;} \\
1.33]\end{array}$ & $\begin{array}{c}0.93 ; 0.71 \\
{[0.29 ;} \\
2.86]\end{array}$ & $\begin{array}{c}0.84 ; 0.80 \\
{[0.33 ;} \\
1.33]\end{array}$ & NS \\
\hline Expertise & $\begin{array}{c}0.92 ; 0.74 \\
{[0.05 ; 7.50]}\end{array}$ & $\begin{array}{c}0.95 ; 0.86 \\
{[0.06 ; 3.33]}\end{array}$ & $\begin{array}{c}0.82 ; 0.69 \\
{[0.05 ; 3.11]}\end{array}$ & $\begin{array}{c}0.95 ; 0.86 \\
{[0.06 ; 3.33]}\end{array}$ & $<.05$ & $\begin{array}{c}0.93 ; 0.68 \\
{[0.33 ;} \\
2.07]\end{array}$ & $\begin{array}{c}0.68 ; 0.47 \\
{[0.05 ;} \\
3.11]\end{array}$ & $\begin{array}{c}0.93 ; 0.68 \\
{[0.33 ;} \\
2.07]\end{array}$ & $<.05$ \\
\hline
\end{tabular}

Les données sont présentées en moyenne ; médiane [étendue].

\section{2. 2. Concernant l'intensité}

Tableau 8. Distribution du rapport de vecteur magnitude de jeu / non-jeu par étape en fonction du rappor de force.

\begin{tabular}{|c|c|c|c|c|c|c|c|c|c|}
\hline \multirow[b]{2}{*}{ Etapes } & \multirow[b]{2}{*}{ Global } & \multicolumn{4}{|c|}{ Rapport de force durant le set } & \multicolumn{4}{|c|}{ Rapport de force en fin de set } \\
\hline & & Dominant & Neutre & Dominé & $P$ & Dominant & Neutre & Dominé & $P$ \\
\hline Structurale & $\begin{array}{c}1.77 ; 1.59 \\
{[0.42 ; 6.02]}\end{array}$ & $\begin{array}{c}1.58 ; 1.46 \\
{[0.42 ; 3.71]}\end{array}$ & $\begin{array}{c}1.80 ; 1.67 \\
{[0.48 ; 6.02]}\end{array}$ & $\begin{array}{c}1.90 ; 1.59 \\
{[0.80 ; 4.69]}\end{array}$ & NS & $\begin{array}{c}1.62 ; 1.66 \\
{[0.68 ; 3.25]}\end{array}$ & $\begin{array}{c}1.81 ; 1.71 \\
{[0.75 ; 3.72]}\end{array}$ & $\begin{array}{c}2.21 ; 1.85 \\
{[1.03 ; 4.66]}\end{array}$ & $<.05$ \\
\hline Fonctionnelle & $\begin{array}{c}2.03 ; 1.86 \\
{[0.11 ; 5.48]}\end{array}$ & $\begin{array}{c}2.01 ; 1.87 \\
{[0.11 ; 4.82]}\end{array}$ & $\begin{array}{c}2.02 ; 1.78 \\
{[0.54 ; 5.18]}\end{array}$ & $\begin{array}{c}2.06 ; 1.92 \\
{[0.65 ; 5.48]}\end{array}$ & NS & $\begin{array}{c}2.25 ; 2.04 \\
{[1.07 ; 3.84]}\end{array}$ & $\begin{array}{l}2.11 ; 1.85 \\
{[0.54 ; 4.83]}\end{array}$ & $\begin{array}{c}2.13 ; 2.06 \\
{[1.09 ; 3.52]}\end{array}$ & NS \\
\hline Technique & $\begin{array}{c}2.25 ; 1.85 \\
{[0.40 ; 13.62]}\end{array}$ & $\begin{array}{c}2.34 ; 1.87 \\
{[0.86 ; 8.26]}\end{array}$ & $\begin{array}{c}2.10 ; 1.82 \\
{[0.40 ; 7.49]}\end{array}$ & $\begin{array}{c}2.24 ; 1.86 \\
{[0.75 ; 7.91]}\end{array}$ & NS & $\begin{array}{c}2.35 ; 1.88 \\
{[1.09 ; 7.07]}\end{array}$ & $\begin{array}{l}2.09 ; 1.80 \\
{[0.65 ; 7.49]}\end{array}$ & $\begin{array}{c}2.19 ; 1.91 \\
{[0.75 ; 7.17]}\end{array}$ & NS \\
\hline Contextuelle & $\begin{array}{c}3.07 ; 2.89 \\
{[0.46 ; 9.07]}\end{array}$ & $\begin{array}{c}3.23 ; 3.08 \\
{[0.70 ; 7.82]}\end{array}$ & $\begin{array}{c}3.05 ; 2.76 \\
{[0.46 ; 9.07]}\end{array}$ & $\begin{array}{c}2.95 ; 2.75 \\
{[0.93 ; 6.91]}\end{array}$ & NS & $\begin{array}{c}3.53 ; 3.40 \\
{[1.35 ; 7.82]}\end{array}$ & $\begin{array}{l}3.14 ; 2.92 \\
{[0.95 ; 9.07]}\end{array}$ & $\begin{array}{c}3.22 ; 2.58 \\
{[1.31 ; 6.38]}\end{array}$ & NS \\
\hline Expertise & $\begin{array}{c}4.88 ; 4.56 \\
{[0.77 ; 11.75]}\end{array}$ & $\begin{array}{c}4.94 ; 4.60 \\
{[1.99 ; 10.01]}\end{array}$ & $\begin{array}{c}4.80 ; 4.45 \\
{[0.77 ; 11.75]}\end{array}$ & $\begin{array}{c}5.06 ; 5.05 \\
{[2.09 ; 13.68]}\end{array}$ & NS & $\begin{array}{c}5.32 ; 4.97 \\
{[1.99 ; 10.01]}\end{array}$ & $\begin{array}{l}4.48 ; 4.32 \\
{[0.88 ; 8.93]}\end{array}$ & $\begin{array}{r}4.96 ; 5.15 \\
{[2.72 ; 7.10]}\end{array}$ & NS \\
\hline
\end{tabular}




\section{eJRIEPS 42 juillet 2017}

L'évolution du rapport de force ou le moment du match n'ont une incidence sur le rythme de jeu qu'à l'étape structurale (1) et à l'étape d'expertise (5).

Aux étapes fonctionnelle (2), technique (3) et contextuelle (4), sur le set global, le rapport temps de jeu / temps de non-jeu ainsi que le rapport VM jeu / VM non-jeu ne sont pas significativement différents entre les trois rapports de force. II en est de même si on isole les données de la fin du set.

A l'étape structurale, on ne note pas de différence par rapport aux autres étapes en termes de durée, par contre, au plan de l'intensité, en fin de set, on observe une différence significative : le rapport VM jeu / VM non-jeu est, à rapport de force neutre, en moyenne de 1.81, à rapport de force dominant de 1.62 et à rapport de force dominé de 2.21. Ainsi le joueur s'agite davantage dans le non-jeu quand il domine et beaucoup moins quand il est dominé en fin de set.

A l'étape d'expertise, sur le set global, le rapport temps de jeu / temps de non-jeu est en moyenne significativement différent entre les trois rapports de force $(0,95$ quand le joueur domine ; 0,82 à rapport de force neutre ; 0,95 quand le joueur est dominé). En fin de set, on observe la même relation (0,93 quand le joueur domine; 0,68 à rapport de force neutre ; 0,93 quand le joueur est dominé). Ainsi, l'expert temporise relativement quand le rapport de force est neutre, par contre il diminue le temps de non-jeu quand le rapport de force évolue (en sa faveur ou en sa défaveur).

Au plan de l'intensité, le rapport VM jeu / VM non-jeu n'est pas significativement différent entre les trois rapports de force. II en est de même si on isole les données de la fin du set.

\section{Discussion}

Afin d'apporter notre contribution aux études récentes (Phomsoupha \& Laffaye, 2014) qui s'intéressent à la question du temps en badminton uniquement chez l'expert, comme si ce sujet lui était exclusivement réservé, nous souhaitions investiguer cette question du débutant à l'expert à l'aide d'un modèle conatif (Bui-Xuân, 1998), matérialisant l'expérience en badminton sous formes d'étapes. Notre hypothèse principale était que le niveau d'expertise avait un effet sur la structure temporelle du set.

De plus, contrairement aux études précitées (Abia'n et al., 2014, Abian- Vicen et al., 2013; Cabello \& Lees, 2004; Cabello Manrique \& González-Badillo, 2003; Chen \& Chen, 2008, 2011; Faude et al., 2007; Ming, Keong, \& Ghosh, 2008), nous souhaitions ici dépasser une approche descriptive (Laffaye et al., 2015) en nous inscrivant dans une perspective plus complexe. D'une part, nous souhaitions mettre en relation ce qui se passe dans le jeu 


\section{eJRIEPS 42 juillet 2017}

avec ce qui se passe dans le non-jeu dans une perspective dynamique. En effet, la durée du non- jeu ne peut s'appréhender ex nihilo mais bien en relation étroite avec la durée de la phase de jeu qui précède. C'est pourquoi les variables retenues dans cette étude étaient des rapports entre jeu et non-jeu. D'autre part, nous ne souhaitions pas appréhender temps et intensité séparément mais bien analyser les deux facteurs concomitamment à travers la notion de rythme de jeu: Nous avons donc défini la dynamique temporelle du set (ou rythme du set) comme la combinaison des différentiels de durée et d'intensité entre les périodes de jeu et de non-jeu.

A la lueur de nos résultats, notre hypothèse principale est vérifiée : notre étude montre qu'en fonction des prévalences conatives, la structuration du ratio exercice/repos en badminton ou rythme du set a une spécificité. A l'étape 1 , le joueur ne prend relativement que peu son temps dans les phases de non-jeu, de plus il s'agite relativement beaucoup. A l'étape 2, le rapport au temps n'évolue pas par rapport à l'étape précédente, par contre le joueur s'agite moins dans les phases de non-jeu. A l'étape 3, le rapport au temps ainsi que le différentiel d'intensité entre jeu et non-jeu n'évolue pas par rapport à l'étape précédente. En revanche à l'étape 4, le joueur accélère le tempo mais s'agite relativement beaucoup moins dans le non-jeu. Enfin, à l'étape d'expertise, le joueur temporise davantage par rapport à l'étape précédente et s'agite beaucoup moins dans le non-jeu.

On note que c'est bien à l'étape d'expertise qu'il y a une hausse significative de la durée du non-jeu. Ce résultat est en phase avec les recherches qui montrent que l'expertise se caractérise par une gestion temporelle du set (O’ Donoghue \& Ingram, 2001). Néanmoins nos résultats en termes de corrélation entre temps de jeu et non-jeu apportent une nuance de taille car ils montrent qu'il n'y a pas de corrélation entre la durée du non-jeu et la période de jeu qui précède pour l'étape d'expertise et ce, même si on différencie les durées de points. Ces résultats remettent en cause les conclusions de Cabello et al. (2003), déjà nuancés par Laffaye et al. (2015) et laissent à penser que la composante énergétique n'est pas suffisante pour expliquer la nature du non-jeu chez l'expert. La durée du non-jeu est sûrement davantage liée au contexte du jeu et donc à une dimension tactique.

Justement, cette étude visait aussi à aborder la notion de rythme, en tenant compte des variations de contextes de jeu. II s'agissait de voir si au sein d'une même étape le rythme du set évoluait en fonction de l'état du rapport de force et/ou en fonction du moment du match. 


\section{eJRIEPS 42 juillet 2017}

Concernant les étapes 2, 3 et 4, on ne note pas de différences significatives de rythmes en fonction des rapports de force (neutre, favorable, défavorable) que ce soit sur le set global ou en fin de set. Par contre, à l'étape 1, on note, qu'en fin de set, au plan de l'intensité, le rapport VM jeu / VM non-jeu est significativement plus faible quand le joueur domine et inversement plus fort quand il est dominé. A l'étape d'expertise, on note une influence majeure de l'état du rapport de force mais aussi du moment de set sur la durée des phases de non-jeu ce qui va dans le sens des réserves émises par Laffaye et al. (2015) concernant les résultats de Cabello et al. (2003) sur la corrélation entre la durée des périodes de non- jeu et la durée des périodes de jeu qui précédent. L'hypothèse tactique est donc bien à mettre à côté de l'hypothèse physiologique pour expliquer les variations dans les durées de non-jeu par rapport à la phase de jeu qui précède.

En définitive, notre étude nous amène aux conclusions suivantes.

A l'étape 1 (structurale), le rythme du set est globalement soutenu, le joueur se mobilisant quasiment autant en non-jeu qu'en jeu. Nous pourrions qualifier ce badiste de « spectateur impatient ». La préoccupation de récupération est ici inexistante. Le rythme s'accélère avec l'évolution du score en fin de set et ne baisse que dans un seul contexte : quand le joueur est dominé en fin de set. Notre hypothèse ici est que l'émotion du point marqué guide le rythme du match en fin de set : l'émotion positive du point gagné guide le rythme à la hausse ; l'émotion négative du point perdu guide le tempo à la baisse. Ce résultat tend à conforter le qualificatif d' " émotionnel » que Bui-Xuân $(1993,1998)$ a donné à cette étape.

A l'étape 2 , le rythme général du set baisse. Si le temps de non-jeu ne diminue pas de manière relative par rapport à l'étape précédente, la quantité de mouvement dans la phase de non-jeu diminue significativement. Néanmoins on n'observe pas de variation du rythme malgré les variations de rapport de force. II en est de même en fin de set. A cette étape, la préoccupation de récupération apparaît mais elle reste formelle. L' « alternateur » a intégré la fonction de récupération du non-jeu (un temps faible) mais celle-ci est indifférenciée en fonction du rapport de force et du moment du match.

A l'étape 3, on ne note pas d'évolution du rythme du set par rapport à l'étape précédente. La nature de la récupération se différencie en étape 4. Si le joueur s'agite encore moins pendant les périodes de non-jeu, la durée de celles-ci diminue significativement : le joueur accélère le tempo. Le joueur a compris qu'on pouvait récupérer tout en épuisant l'adversaire. Par contre on n'observe pas de variation du rythme en fonction de l'évolution 


\section{eJRIEPS 42 juillet 2017}

du rapport de force ou du moment du set. Le joueur d'étape 4, tel un « rouleaucompresseur » impose un rythme mais uniforme.

A l'étape 5, globalement, le joueur temporise à nouveau contrairement à l'étape précédente et s'agite beaucoup moins encore dans les phases de non-jeu. Par contre il accélère le tempo quand il domine ou quand il est dominé tout en s'agitant très peu dans les phases de non-jeu. On observe la même tendance en fin de set. A cette étape, la préoccupation de récupération s'inscrit dans une réelle dynamique de set. Les notions de « temps fort / temps faible » deviennent l'outil du joueur expert qui tel un « meneur » cherche à ne pas subir le rythme adverse (à rapport de force neutre) tout en imposant le sien aux moments opportuns (mettre l'adversaire sous pression quand le rapport de force évolue). Cette «gestion de l'engagement en cours de set » lui permet de mettre encore du rythme à la fin, les cinq derniers points étant décisifs. A cette étape le joueur dispose de son rythme, rythme qu'il différencie en fonction des contextes. On peut ici parler de dynamique de set, dans laquelle le non-jeu est au service de la gestion du score. Si Laffaye et al. (2015) évoquait l'aspect tactique et notamment la discussion avec le coach pour justifier un temps de non-jeu long même après un point court, nos résultats tendent à montrer que l'expert temporise certes pour des raisons tactiques, mais qu'il peut accélérer aussi pour ces mêmes raisons.

\section{Conséquences pédagogiques}

En pédagogie, l'utilisation d'un jeu au temps, le même pour tous (et un temps souvent très court pour des scolaires), est à discuter puisque le rythme du jeu, c'est-à-dire la structuration du ratio exercice/repos n'est pas uniforme mais varie selon le niveau d'expertise. L'intervenant légitime souvent ces pratiques au temps en mettant en avant sa responsabilité d'offrir un temps de travail moteur égal pour l'ensemble des élèves (Leveau, 2007). Si l'intention est louable, la montante-descendante au temps majoritairement utilisée en EPS semble à terme démobiliser les élèves, notamment les filles, au fur et à mesure que la scolarité augmente (Deslaurier, 2007 ; Dieu et al., 2016). Par ailleurs, le jeu « au temps », systématique et indifférencié, est une pratique inéquitable dans le cadre d'évaluations de type recueils statistiques, basées sur le nombre de points joués. En effet, comme le rapport au temps diffère selon les étapes, un grand débutant aura de facto un nombre de points joués plus faible qu'un joueur de l'étape 2 ou 3, puisqu'il fait significativement des échanges plus longs. 


\section{eJRIEPS 42 juillet 2017}

C'est pourquoi nous proposons de substituer à une responsabilité organisationnelle, une responsabilité didactique qui consiste à respecter la mobilisation des élèves, à savoir le sens profond qu'ils confèrent à leurs actions en fonction de leur prévalence conative. En effet, pour respecter la valeur de la chose placée sous responsabilité (Jonas, 1979), ici l'expérience vécue de l'élève, le respect de la mobilisation, c'est-à-dire la spécificité la structuration du ratio exercice/repos, doit supplanter la simple volonté de mise en action des élèves.

Dès lors, le jeu au temps est à manier avec précaution dans les stratégies d'enseignement du badminton sous peine d'imposer un rythme en discordance (Bui-Xuân, 1998) avec l'étape conative du joueur et inhiber sa progression dans le curriculum.

Cette pratique pédagogique peut être employée pour les joueurs de l'étape 1 (qui n'ont pas encore intégré la fonction du non jeu) si ce temps est long... En effet, ressentir la fatigue va les amener à se poser la question « comment gagner sans être épuisé ? » et ainsi favoriser le passage à l'étape 2 qui consiste à diminuer la quantité de mouvements pendant les périodes de non-jeu.

A partir de l'étape 2, le jeu au score doit reprendre le pas puisque le joueur a perçu l'utilité globale du non-jeu. Le jeu au temps en l'obligeant à enchaîner très vite pour ne pas perdre la course viendrait contrecarrer cette logique naissante. Enfin, pour accéder à l'expertise, nous préconisons de jouer sur des scores très courts (simuler une fin de set) ou avec des handicaps (faire varier le rapport de force) afin de construire les notions de temps forts où le joueur met du rythme pour jouer les points importants.

Nos résultats doivent néanmoins être nuancés. En effet, la distinction d'âge et de sexe en tant que variables influençant la quantité de mouvement n'a pas été retenue. Or, certains auteurs ont montré que le sexe avait une influence sur l'intensité du match, en tennis notamment (Fernandez, Mendez-Villanueva \& Pluim, 2006). Par ailleurs, dans cette étude n'a pas été prise en compte la notion de style de jeu par absence de questionnement sur la stratégie du joueur. Ainsi, en perspective, la réalisation d'entretiens pour affiner l'analyse tactique du set serait heuristique. Enfin, l'analyse n'a porté que sur un set, étendre l'exploration sur un match complet permettrait d'appréhender plus finement le contexte de jeu et son évolution sur le long terme. 


\section{eJRIEPS 42 juillet 2017}

\section{Conclusion}

Cette étude portait sur 40 joueurs de badminton de tous niveaux qui se sont opposés en contexte de match équipés d'un accéléromètre triaxial. Ces joueurs ont été classés en fonction de leur expérience du badminton dans une des cinq étapes du curriculum conatif (Bui-Xuân, 1998 ; Vanhelst, 2012a). Notre étude montre, qu'en fonction des prévalences conatives, la structuration du ratio exercice/repos en badminton ou rythme du set a une spécificité. De plus, au sein même de certaines étapes, pour les experts et les grands débutants, on observe une variation de ces facteurs en fonction de l'évolution du rapport de force et / ou du moment du match.

II y a donc des rythmes ou temporalités qui se redéfinissent en fonction des intentions de jeu ou des contextes de jeu.

En pédagogie, l'utilisation du jeu « au temps » fait qu'une temporalité unique est subie par l'ensemble des joueurs. C'est davantage la spécificité du jeu (expérience du joueur, état du rapport de force, moment du match) qui devrait orienter le choix didactique entre jeu au temps / jeu au point afin d'envisager la temporalité de manière plus responsable.

\section{Bibliographie}

Abia'n, P., Castanedo A., Feng X. Q., Sampedro J., \& Abian-Vicen J. (2014). Notational comparison of men's singles badminton matches between Olympic Games in Beijing and London. International Journal of Performance Analysis in Sport, 14, 4253.

Abian-Vicen, J., Castanedo, A., Abia'n, P., \& Sampedro, J. (2013) Temporal and notational comparison of badminton matches between men's singles and women's singles. International Journal of Performance Analysis in Sport, 2, 310-320.

Alain, C., \& Proteau, L. (1978). Etude des variables relatives au traitement de l'information dans les sports de raquette. Canadian Journal of Applied Sport Sciences, 3, 240244.

Andréani, P. (1999). Badminton, la frappe longue : repères techniques et pédagogiques. Revue EP.S, 279, 58-59.

Bailey, R. C., Olson J., Pepper S. L., Porszasz J., Barstow T. J., \& Cooper D. M. (1995). The level and tempo of children's physical activities: an observational study. Medicine and Science in Sports and Exercise, 27(7), 1033-41. 


\section{eJRIEPS 42 juillet 2017}

Bui-Xuan, G. (1994).Une modélisation du procès pédagogique. In J. P. Clement \& M. Herr (Eds.), L'identité de l'éducation physique scolaire au XXe siècle (p.77-90). Clermont-Ferrand : AFRAPS.

Bui-Xuân, G. (1998). Le corps mobilisé. HDR. Montpellier : Université de Montpellier 1.

Cabello, D., \& Lees, A. (2004). Temporal and physiological characteristics of elite womens and mens singles badminton. International Journal of Applied Sports Sciences, 16(2), 1-12.

Cabello Manrique D., \& Gonzalez-Badillo J.-J. (2003). Analysis of the Caracteristics of Competitive Badminton. British Journal of Sport and Medicine, 37(1), 62-6.

Chen, H. -L., \& Chen, T. C. (2008). Temporal structure comparison of the new and conventional scoring systems for men's badminton singles in Taiwan. Journal of Exercise Science and Fitness, 6(1), 34-43.

Chen, H. -L., \& Chen, T. C. (2011). Physiological and notational comparison of new and old scoring systems of singles matches in men's badminton. Asian Journal of Physical Education and Recreation, 17(1), 6-17.

Chen, K. Y., \& Bassett, D. R., Jr. (2005). The technology of accelerometry-based activity monitors: Current and future. Medicine and Science in Sports and Exercise, 37, 490-500.

Chu, E. Y., McManus, A. M., \& Yu, C. C. (2007). Calibration of the RT3 accelerometer for ambulation and non-ambulation in children. Medicine and Science in Sports and Exercise, 39, 2085-2091.

Couartou, G. \& Grunenfelder, F. (1992). Badminton, de l'école... aux associations. Paris : Editions revue EPS.

Craig, C. L., Marshall, A. L., Sjostrom, M., Bauman, A. E., Booth, M. L., Ainsworth, B. E., Pratt, M., Ekelund, U., Yngve, A., Sallis, J. F., \& Oja, P. (2003). International Physical Activity Questionnaire: 12-country reliability and validity. Medicine and Science in Sports and Exercise, 35, 1381-95.

Deconinck, O., \& Fontaine, E. (2002). Impliquer l'élève dans un projet : illustrations en volley-ball et badminton. Revue EP.S, 295, 57-62.

De Jonge, L., DeLany, J. P., Nguyen, T., Howard, J., Hadley, E. C., Redman, L. M. \& Ravussin, E. (2007). Validation study of energy expenditure and intake during calorie restriction using doubly labelled water and changes in body composition. The American Journal of Clinical Nutrition, 85, 73-9. 


\section{eJRIEPS 42 juillet 2017}

Descamps, O. (2008). Badminton : la montante-descendante : situation de référence et/ou d'apprentissage. Revue EP.S, 332, 39-42.

Dieu, O. (2010). Badminton : l'éternel débutant. Revue EP.S, 343, 10-13.

Dieu, O. (2012). Expérience corporelle et sens du mouvement : matérialisation via l'actimétrie du « contexte altéré par l'action » dans l'évolution du joueur de badminton. STAPS, 98, 49-65.

Dieu, O. (2015). Corrélation entre orientation tactique du jeu et quantité d'activité physique en badminton. eJRIEPS, 36, 99-120.

Dieu, O., Joing, I., Drumez, E. (2016). Teaching Practices and Symbolic Violence: The Case of Racket Sports at School. STAPS, 112, 85-98.

Estrabaud, P., Murignieux, E. \& Tixier-Viriciel C., (2000). Badminton au baccalauréat : un exemple pratique d'évaluation. Revue EP.S, 284, 23-25.

Fahimi, F. \& Vaezmousavi, M. (2011). Physiological patterning of short badminton serve: a psychophysiological perspective to vigilance and arousal. World Applied Science Journal 12(3), 347-53.

Faude, O., Meyer, T., Rosenberger, F., Fries, M., Huber, G. \& Kindermann, W. (2007). Physiological characteristics of badminton match play. European Journal of Applied Physiology, 100 (4), 479-85.

Ferly, B. \& Papelier, G. (2003). Enseigner le Badminton en milieu scolaire. Les cahiers Actio.

Fernandez, J., Mendez-Villanueva, A. \& Pluim, B. M. (2006). Intensity of tennis match play. British Journal of Sports and Medicine, 40(5), 387-91.

Gauvin J. (1995). Badminton, les «zones - cibles ». Revue EP.S, 252, 13-15.

Jonas, H. (1979). Le principe responsabilité.

Laffaye, G. (2006). Evaluer la performance : les défis. Revue EP.S, 322, 23-26.

Laffaye, G. (2011). Comprendre et progresser en badminton. Magny-Les-Hameaux, France : Chiron.

Laffaye, G., Phomsoupha, M. \& Dor, F. (2015). Changes in the Game Characteristics of a Badminton Match: A Longitudinal Study through the Olympic Game Finals Analysis in Men's Singles. Journal of Sports Science and Medicine, 14, 584-90.

Leenders, N.Y.J.M., Sherman, W. M. \& Nagaraja, H. N. (2000). Comparisons of four methods of estimating physical activity in adult women. Medicine and Science in Sports and Exercise, 32, 1320-26. 


\section{eJRIEPS 42 juillet 2017}

Lees, A. (2003). Science and the major racket sports: a review. Journal of Sports Sciences, 21, 707-32.

Leveau, C. (2000). Notion de pression temporelle dans la relation duelle : un exemple en badminton. In E. Louis (Ed.), Sports de raquette, entre pratiques et théories. Dossier EPS n53 (pp. 43-59). Paris : Editions Revue EP. S.

Leveau, C. (2007). Le Badminton en situation, « observer et intervenir ». Paris : Editions Revue EPS.

Liddle, D. \& O' Donoghue, P. (1998). Notational analysis of rallies in European circuit badminton. In A. Lees, M. Hughes, T. Reilly \& I. Maynard (Eds). Science and Racket Sports II (pp. 275-282). London: E \& FN Spon.

Limouzin, P. (1994). APS éducative, le badminton. Hyper, 189, 15-19.

Limouzin, P. \& Wright, I. (2000). Badminton, vers le haut niveau. Paris : INSEP.

Mérard, M. \& Ventou, P. (1996). Badminton, le rapport de force : trait d'union entre la théorie et la pratique. Revue EPS, 259, 17-20.

Ming, C. L., Keong, C. C. \& Ghosh, A. K. (2008) Time motion and notational analysis of 21 point and 15 point badminton match play. International Journal of Sports Science and Engineering, 2(4), 216-22.

O' Donoghue, P. (2000). Notational analysis of rallies in European Club Championship Badminton. In M. Hughes \& F. Tavares (Eds). Notational Analysis of Sports IV (pp. 225-229). Porto: FCDEF.

O' Donoghue, P., Ingram, B. (2001). A notational analysis of elite tennis strategy. Journal of Sport Science, 19 (2): 107-15.

Phomsoupha, M. \&, Laffaye, G. (2014). The Science of Badminton: Game Characteristics, Anthropometry, Physiology, Visual Fitness and Biomechanics. Sports Medicine, 45, 473-95.

Pizzinato, A. (1993). Le tennis. Nancy : Presses universitaires de Nancy.

Pritchard, S., Hughes, M. \& Evans, S. (2001). Rule changes in elite badminton. In Hughes, M. and I. Franks, I. (Eds). Proceedings of pass.com Conference (pp. 213-222). Cardiff: CPA, UWIC.

Preuvot, D. (2000). Sports de raquettes : entre pratique, programmes et théorie. In Eric Louis (Ed.), Sports de raquette, entre pratiques et théories. Dossier EPS n`53 (pp. 89-99). Ed. Revue EP. S. 


\section{eJRIEPS 42 juillet 2017}

Rowlands, A. V., Thomas, W. M., Eston, R. G. \& Topping, R. (2004). Validation of the RT3 Triaxial accelerometer for the assessment of physical activity. Medicine and Science in Sports and Exercise, 36, 518-24.

Sève, C. (2000). Dynamique et signification de l'activité des pongistes en match. In E. Louis (Ed.), Sports de raquette, entre pratiques et théories. Dossier EPS n53 (pp. 89-99). Paris : Editions Revue EP. S.

Silva, P., Santiago, C., Reis, L. P., Sousa, A., Mota, J. \& Welk, G. (2015). Assessing physical activity intensity by video analysis. Physiological Measurement, 36, 103746.

Thibaut, R. (1993). Le badminton. Montréal : Gaëtan Morin Editeur.

Treuth, M. S., Schmitz, K., Catellier, D. J., McMurray, R. G., Murray, D. M., Almeida, M. J., Going, S., Norman J. E \& Pate R. (2004b). Defining accelerometer thresholds for activity intensities in adolescent girls. Medicine and Science in Sports and Exercise, 36, 1259-66.

Vanhelst, J., Zunquin, G., Theunynck, D., Mikulovic, J., Bui-Xuan, G. \& Béghin, L. (2009). Equivalence of accelerometer data for walking and running: Treadmill versus on land. Journal of Sports Sciences, 27 (7), 669-75.

Vanhelst J., Béghin, L., Rasoamanana, P., Theunynck, D., Meskini, T., Iliescu, C., Duhamel, A., Turck, D., \& Gottrand, F. (2010). Calibration of the RT3 accelerometer for various patterns of physical activity in children and adolescents. Journal of Sports Sciences, 28(4), 381-87.

Vanhelst, J., Beghin, L., Fardy, P., Bui-Xuân, G. \& Mikulovic, J. (2012a). A conative educational model for an intervention program in obese youth. BMC Public health, $12,416$.

Vanhelst, J., Beghin, L., Duhamel, A., Bergman, P., Sjöström, M., Gottrand, F. (2012b) Comparison of uniaxial and triaxial accelerometry in the assessment of physical activity among adolescents under free-living conditions: the HELENA study. BMC Medical Research Methodology, 12-26.

Vaufreydaz, C. (2000). Classe de seconde, exemple d'application en badminton. Revue EP.S, 281, 23-25. 